\title{
Dissociation of High-Pressure Solid Molecular Hydrogen: A Quantum Monte Carlo and Anharmonic Vibrational Study
}

\author{
Sam Azadi, ${ }^{1, *}$ Bartomeu Monserrat, ${ }^{2, \dagger}$ W. M. C. Foulkes, ${ }^{1}$ and R. J. Needs ${ }^{2}$ \\ ${ }^{1}$ Thomas Young Centre and Department of Physics, Imperial College London, London SW7 2AZ, United Kingdom \\ ${ }^{2}$ TCM Group, Cavendish Laboratory, University of Cambridge, J. J. Thomson Avenue, Cambridge CB3 OHE, United Kingdom
}

(Received 14 November 2013; published 22 April 2014)

\begin{abstract}
A theoretical study is reported of the molecular-to-atomic transition in solid hydrogen at high pressure. We use the diffusion quantum Monte Carlo method to calculate the static lattice energies of the competing phases and a density-functional-theory-based vibrational self-consistent field method to calculate anharmonic vibrational properties. We find a small but significant contribution to the vibrational energy from anharmonicity. A transition from the molecular $\mathrm{Cmca}-12$ direct to the atomic $I 4_{1} /$ amd phase is found at $374 \mathrm{GPa}$. The vibrational contribution lowers the transition pressure by $91 \mathrm{GPa}$. The dissociation pressure is not very sensitive to the isotopic composition. Our results suggest that quantum melting occurs at finite temperature.
\end{abstract}

DOI: 10.1103/PhysRevLett.112.165501

In 1935, Wigner and Huntington [1] predicted that solid molecular hydrogen would dissociate at high pressure to form a metallic atomic solid. The properties of atomic hydrogen have fascinated high-pressure scientists and astrophysicists ever since [2,3]. Various exotic predictions have been made, such as the stability of atomic metallic hydrogen in a superfluid state or as a room-temperature superconductor [4-6], but neither an insulator-to-metal transition nor a molecular-to-atomic transition has yet been observed unambiguously at low temperatures.

The nature of hydrogen at high pressure is currently the subject of intense interest. Experimental studies of hydrogen and deuterium have been performed up to pressures above $300 \mathrm{GPa}$ using static diamond-anvil-cell (DAC) techniques [3,7-12]. A new high-pressure phase IV of hydrogen and deuterium was recently observed, which is believed to consist of alternate layers of strongly bonded molecules and weakly bonded graphenelike sheets $[8,13]$. The precise pressures achieved in these experiments may have been overestimated and are still controversial [14]. Even more controversial is the suggestion that conductive dense hydrogen has been produced in room-temperature experiments [7]. However, the discovery of weak bonding in phase IV suggests that static DAC experiments could probe the conditions under which the full molecular dissociation of hydrogen and deuterium take place. The results of our work corroborate this suggestion.

We have studied hydrogen in the pressure range 300$650 \mathrm{GPa}$, within which the transition from molecular to

Published by the American Physical Society under the terms of the Creative Commons Attribution 3.0 License. Further distribution of this work must maintain attribution to the author(s) and the published article's title, journal citation, and DOI.
PACS numbers: $81.40 . \mathrm{Vw}, 02.70 . \mathrm{Ss}, 63.20 . \mathrm{dk}, 64.70 . \mathrm{K}-$

atomic structures is thought likely to occur. The most important contribution to the structural energy is the static lattice energy. The energy differences between competing phases in hydrogen are small, and a very accurate description of the electronic energy is required to resolve them. We have therefore calculated static lattice energies using the diffusion quantum Monte Carlo (DMC) method, which is the most accurate method known for evaluating the energies of large assemblies of interacting quantum particles [15-18].

Experimental measurements [19-21] and classical molecular dynamics simulations using density-functionaltheory (DFT) methods [22,23] suggest that the melting temperature of hydrogen increases with pressure and reaches a maximum value of roughly $1000 \mathrm{~K}$ at a pressure in the region of $100 \mathrm{GPa}$, whereafter it declines with pressure. Pathintegral molecular dynamics simulations have suggested that the inclusion of the zero-point (ZP) energy of the protons reduces the melting temperature to about $160 \mathrm{~K}$ at $500 \mathrm{GPa}$ and $100 \mathrm{~K}$ at $800 \mathrm{GPa}$ [24]. This suggests that the interatomic bonding becomes very weak at these pressures, and anharmonic effects could become important.

We have performed vibrational self-consistent field (VSCF) calculations within DFT to calculate the anharmonic vibrational ZP energies [25]. We used the Perdew-BurkeErnzerhof (PBE) generalized gradient approximation density functional, which is well suited for very high-pressure studies, as the charge density is more uniform than at low densities, and it obeys the uniform limit and gives a good account of the linear response of the electron gas to an external potential [26].

Static lattice DFT calculations using ab initio random structure searching [27] indicate that there are three energetically competitive structures in the range of interest. The molecular $\mathrm{Cmca}-12$ phase is insulating up to $373 \mathrm{GPa}$ in the $G W$ approximation [28], although proton zero-point 
and finite-temperature effects are expected to lower the metallization pressure [29]. The molecular $\mathrm{Cmca}-4$ phase and the atomic phase of $I 4_{1} /$ amd symmetry (the structure of Cs-IV) are both metallic [30-32]. DFT with the PBE functional predicts that $\mathrm{Cmca}-12$ is stable up to $385 \mathrm{GPa}$, Cmca-4 is stable in the range 385-490 GPa, and I4 $/$ /amd is stable from $490 \mathrm{GPa}$ up to pressures beyond $1 \mathrm{TPa}$.

DFT studies of high-pressure phases of hydrogen have been performed using several approximate density functionals $[13,24,29,33,34]$, and a significant dependence of the results on the functional has been noted. The enthalpy differences between phases are so small that changes of only a few meV per proton can make a noticeable difference to the phase diagram. It is therefore important to use an accurate approach for calculating the energies of the competing phases. We have chosen to use the DMC method $[15,16]$ to calculate the static lattice energies. This method solves the many-electron Schrödinger equation, is, in principle, exact for the ground states of the hydrogen atom and molecule, and rigorously excludes self-interaction errors. It is likely that the DMC method provides a considerably more accurate description of the energetics of hydrogen than the currently available exchange-correlation density functionals.

We used the CASINO code [35] to perform fixed-node DMC simulations with a trial wave function of the Slater-Jastrow (SJ) form

$$
\Psi_{\mathrm{SJ}}(\mathbf{R})=\exp [J(\mathbf{R})] \operatorname{det}\left[\psi_{n}\left(\mathbf{r}_{i}^{\uparrow}\right)\right] \operatorname{det}\left[\psi_{n}\left(\mathbf{r}_{j}^{\downarrow}\right)\right],
$$

where $\mathbf{R}$ is a $3 N$-dimensional vector of the positions of the $N$ electrons, $\mathbf{r}_{i}^{\uparrow}$ is the position of the $i$ th spin-up electron, $\mathbf{r}_{j}^{\downarrow}$ is the position of the $j$ th spin-down electron, $\exp [J(\mathbf{R})]$ is a Jastrow factor, and $\operatorname{det}\left[\psi_{n}\left(\mathbf{r}_{i}^{\uparrow}\right)\right]$ and $\operatorname{det}\left[\psi_{n}\left(\mathbf{r}_{j}^{\downarrow}\right)\right]$ are Slater determinants of spin-up and spin-down one-electron orbitals. These orbitals were obtained from DFT calculations performed with the plane-wave-based QUANTUM ESPRESSO code [36], employing a norm-conserving pseudopotential constructed within DFT using the local density approximation (LDA) exchange-correlation functional. The choice of exchange-correlation functional used to generate the orbitals has almost no effect on the DMC energies of solid hydrogen phases $[28,37]$. Earlier work also suggests that using a pseudopotential has only a small impact on results for high-pressure solid hydrogen [32]. We chose a very large basis-set energy cutoff of 300 Ry to approach the complete basis-set limit [38], as detailed in the Supplemental Material [39]. The plane-wave orbitals were transformed into a localized "blip" polynomial basis [40]. Our Jastrow factor consists of polynomial one-body electron-nucleus and twobody electron-electron terms, the parameters of which were optimized by minimizing the variance of the local energy at the variational Monte Carlo level [41,42]. The quantum Monte Carlo calculations were performed with simulation cells containing $N=128$ protons. We used twist-averaged boundary conditions with 24 randomly chosen twists to reduce the single-particle finite-size effects [43]. We have corrected our results for the effects of using finite simulation cells, employing the approach described in Refs. $[44,45]$. The residual finite-size effects are estimated to lead to errors in the enthalpy differences between phases of less than $5 \mathrm{meV}$ per proton. The finite-size corrections are detailed in the Supplemental Material [39]. The statistical errors in our data are smaller than the reported accuracy [39].

The enthalpy was evaluated by fitting a polynomial to the finite-size-corrected DMC energy as a function of volume and differentiating the fit. The resulting enthalpypressure relations are shown in the upper plot of Fig. 1. At the static lattice level, we find a transition from $\mathrm{Cmca}-12$ to Cmca-4 at $431 \mathrm{GPa}$ and a transition from the molecular Cmca-4 to the atomic I4 $/$ amd structure at about $465 \mathrm{GPa}$. DMC calculations predict that the Cmca-4 phase is significantly less stable than in the PBE DFT.

To explore the accuracy of the DMC results further, we performed calculations with trial wave functions incorporating an inhomogeneous backflow (BF) transformation [46], which modifies the nodal surface of the wave function and can introduce additional correlation effects. The BF
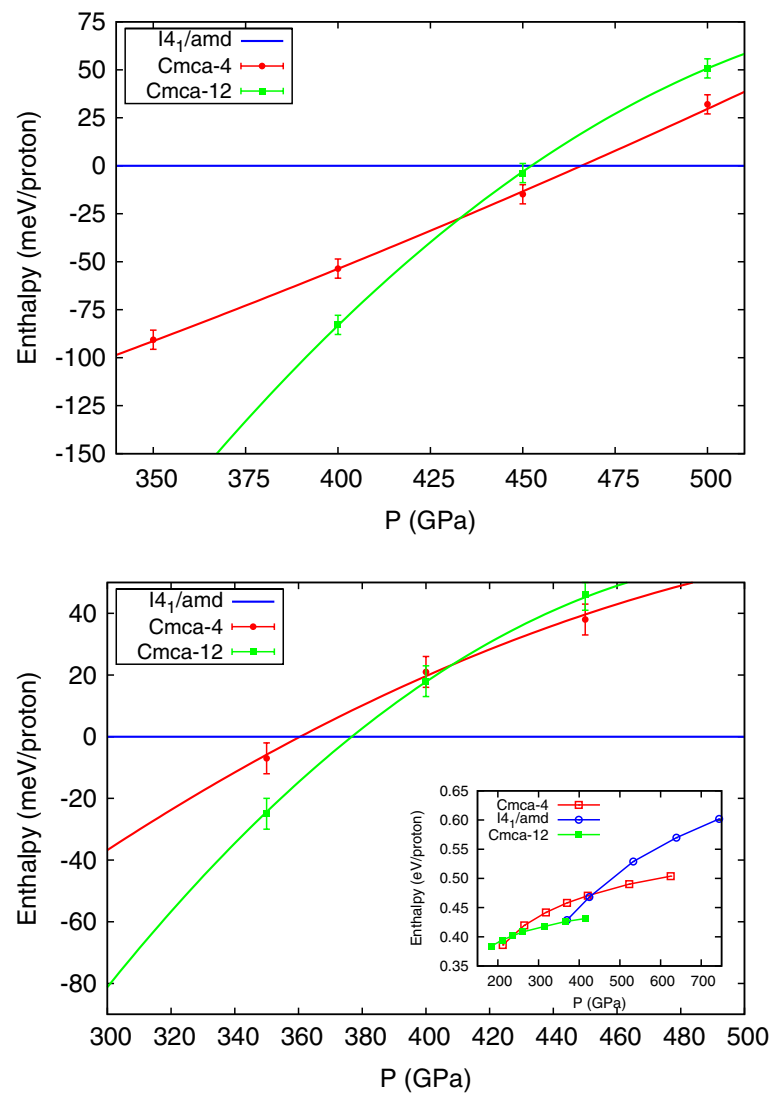

FIG. 1 (color online). Enthalpy as a function of pressure for the Cmca-12, Cmca-4, and I4 $/$ amd phases, relative to the $I 4_{1} /$ amd phase. Top: Static phase diagram from DMC calculations. Bottom: Phase diagram including the ZP enthalpy from the harmonic and anharmonic vibrational calculations. The inset shows the harmonic $\mathrm{ZP}$ enthalpy as a function of pressure. 
wave functions give energies lower than the SJ wave functions by about $19-13 \mathrm{meV}$ per proton with increasing density for $\mathrm{Cmca}-4$, while the $I 4_{1} /$ amd energies were lowered by about $17 \mathrm{meV}$ per proton, approximately independently of density. The energy reduction in the molecular $\mathrm{Cmca}^{-4}$ phase is slightly larger than in the $I 4_{1} /$ amd atomic phase, but the energy reductions of the Cmca-4 and I $4_{1} /$ amd phases are almost the same in the region of the phase transition. We conclude that the introduction of BF correlations does not significantly alter our results. Further details of the effects of BF are described in the Supplemental Material [39].

The above results are based on static lattice calculations in which the vibrational motion of the protons has been neglected. We have also performed calculations of the ZP enthalpy arising from the proton motion using (a) the quasiharmonic approximation and (b) a VSCF approach that enables the calculation of anharmonic vibrational energies [25]. The quasiharmonic phonon calculations were performed with the PBE functional using both the supercell finite displacement method and density-functional perturbation theory as implemented in QUANTUM ESPRESSO [36].

Previous calculations of quasiharmonic proton ZP energies in solid hydrogen have encountered significant numbers of unstable phonon modes [31,32] at high pressures. We found that the Cmca-12 and I4 / amd structures had stable modes at the supercell sizes considered. For $\mathrm{Cmca}-4$, we found a small unstable region around the $\Gamma$ point which was further reduced, but not entirely eliminated, by using cells with up to 256 protons. This small unstable region does not affect our estimates of the ZP energy, as shown in the Supplemental Material [39]. As illustrated in the inset of the lower panel of Fig. 1, the proton ZP enthalpy of all three phases increases with pressure.

Systems of light atoms with weak bonding often exhibit large vibrational amplitudes, which are likely to give rise to anharmonic vibrations. There is evidence for the importance of anharmonicity in hydrogen, especially in the highdensity regime $[2,29]$. Utilizing our recently developed variational VSCF scheme [25,47], we have calculated the anharmonicity of the proton $\mathrm{ZP}$ motion of both the molecular and atomic phases. We use the principal axes approximation to map the Born-Oppenheimer energy surface along independent but anharmonic vibrational modes $[25,48]$ and solve the resulting equations within a VSCF scheme $[25,49]$. We also calculate the contribution from phonon-phonon two-body coupling in the most anharmonic modes to estimate the effects of these terms on the anharmonic vibrational energy [39]. The BornOppenheimer energy surface is mapped within plane-wave DFT using the CASTEP code [50]. By comparing the energies of the highest- and lowest-energy modes with those of the static lattice, we estimate that our choice of computational parameters leads to energy differences between frozen phonon configurations that are converged to within $10^{-4} \mathrm{eV} /$ proton. All calculations were performed with the PBE functional and supercells containing 96 and 108 atoms. Supercell-size convergence tests indicate that the anharmonic ZP energy correction is accurate to within $1 \mathrm{meV} /$ proton for all three phases. Further details of the VSCF calculations are given in the Supplemental Material [39]. We performed calculations for the atomic I $4_{1} /$ amd phase at pressures of $P=400,500$, and $600 \mathrm{GPa}$, obtaining anharmonic corrections of $-7.2,-8.1$, and $-7.3 \mathrm{meV} /$ proton, respectively. Similar calculations for the molecular $\mathrm{Cmca}^{-4}$ phase at pressures of $\mathrm{P}=400$ and $500 \mathrm{GPa}$ give anharmonic corrections of +8.7 and $+8.3 \mathrm{meV} /$ proton, respectively. Calculations at $P=$ $400 \mathrm{GPa}$ for the $\mathrm{Cmca}-12$ structure lead to an anharmonic correction of $+4.0 \mathrm{meV} /$ proton. The anharmonic corrections to the proton $\mathrm{ZP}$ energy lower the energy of the atomic phase and raise the energy of the molecular phases.

As an example of the vibrational properties of the $I 4_{1} /$ amd structure, we plot in Fig. 2 the Born-Oppenheimer energy surface and the corresponding anharmonic wave function density at $P=500 \mathrm{GPa}$ for a $\Gamma$-point optical phonon of the $I 4_{1} /$ amd structure, and a comparison with the harmonic quantities. The $I 4_{1} /$ amd structure can be viewed as a sequence of four stacked planes with square lattices, as shown in the lower panel of Fig. 2. The mode corresponds to an in-plane motion of the protons, where alternate stacked

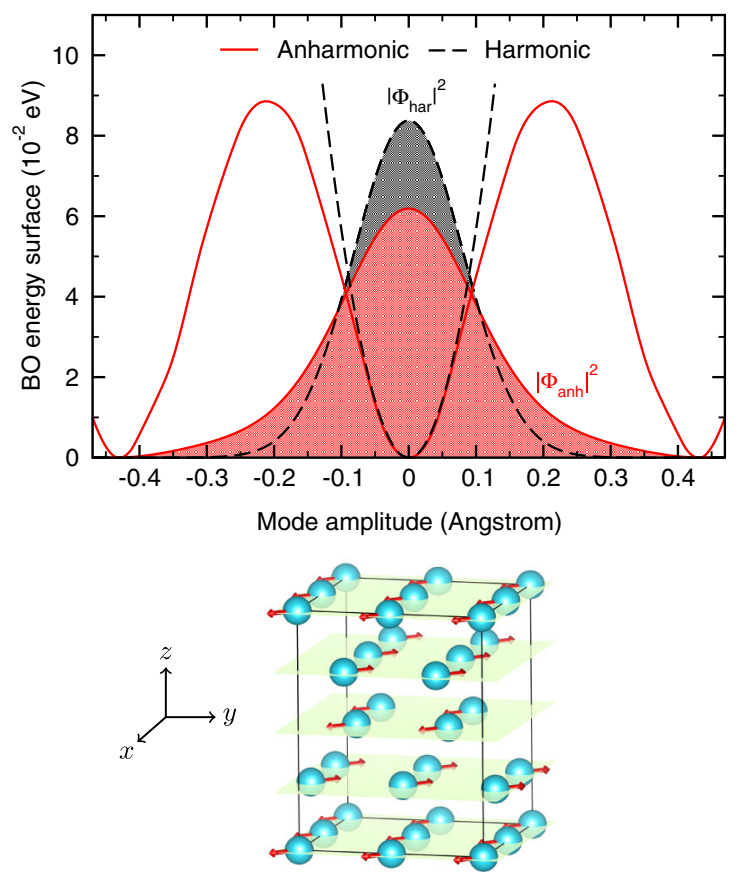

FIG. 2 (color online). Top: Harmonic (dashed black lines) and anharmonic (solid red lines) Born-Oppenheimer (BO) energy surfaces and corresponding wave function densities $\left|\Phi_{\text {har }}\right|^{2}$ and $\left|\Phi_{\text {anh }}\right|^{2}$ for an optical mode at the $\Gamma$ point of the $I 4_{1} /$ amd structure. Bottom: A supercell of the $I 4_{1} /$ amd structure with arrows indicating the proton motion corresponding to the phonon mode in the top figure. Alternate planes move in antiphase. 
planes oscillate in opposite directions. The minima of the anharmonic potential are separated by about $0.427 \AA$ in real space. Adjacent minima correspond to equivalent $I 4_{1} /$ amd structures connected by this in-plane proton motion. This is the mode with the largest anharmonicity in the $I 4_{1} /$ amd structure.

We note that the fermionic nature of the protons has not been taken into account at either the harmonic or anharmonic level. In order to estimate the effects of this approximation, we consider the real space amplitude of the motion of the protons about their equilibrium positions. The root-mean-square atomic amplitude in the $I 4_{1} /$ amd phase at $P=500 \mathrm{GPa}$ is $\sqrt{\left\langle u^{2}\right\rangle}=0.099 \AA$, which is much smaller than the nearest neighbor distance of $a=0.99 \AA$. This indicates a small overlap between the proton wave functions and justifies the neglect of their quantum statistics. The Lindemann criterion [51] for the melting of a solid is usually taken to be $\sqrt{\left\langle u^{2}\right\rangle} / a \gtrsim 0.1$, but for quantum melting, a value of $\sqrt{\left\langle u^{2}\right\rangle} / a \gtrsim 0.25$ is considered more accurate [52-54]. From our data [39], $\sqrt{\left\langle u^{2}\right\rangle} / a=0.1$ at $T=0 \mathrm{~K}$, which is not in the regime of a zero-temperature quantum liquid [6]. The inclusion of quantum statistics would increase the kinetic energy of the liquid and make it even less favorable at zero temperature. The Lindemann criterion applied to the corresponding atomic phase for the heavier deuterium would lead to a higher melting temperature. The Lindemann criterion cannot definitely answer the question of whether the ground-state atomic phase is a metallic solid or a quantum liquid, but it suggests that the melting temperature is higher than $0 \mathrm{~K}$. These conclusions should be compared with recent path-integral molecular dynamics results at $P=$ $500 \mathrm{GPa}$ [24], which suggest a melting temperature of $160 \mathrm{~K}$.

We estimate the dynamical enthalpy as the sum of the static DMC enthalpy and the ZP enthalpy calculated using the quasiharmonic approximation corrected by the VSCF scheme to account for the effects of anharmonicity. The contribution to the total pressure from the ZP motion for the atomic $I 4_{1} /$ amd phase increases with pressure from 25 to $45 \mathrm{GPa}$ over the pressure range of the inset in the lower panel of Fig. 1, while the ZP pressures of the molecular Cmca-4 and $\mathrm{Cmca}-12$ phases increase from 19 to $24 \mathrm{GPa}$ and 10 to $13.5 \mathrm{GPa}$, respectively. As shown in the dynamical phase diagram of Fig. 1, the transition from the Cmca-12 to the atomic I4/ / amd phase occurs at $374 \mathrm{GPa}$, and there is no stability region for the Cmca-4 phase.

At the static level, the phase diagrams of hydrogen and deuterium are identical. At the dynamic level and using the quasiharmonic approximation, the deuterium ZPE is calculated by dividing the hydrogen ZPE by $\sqrt{2}$. As illustrated in the Supplemental Material [39], the dynamical phase diagram of deuterium shows that the molecular-to-atomic phase transition happens at a pressure of $390 \mathrm{GPa}$, also through a structural transformation from molecular Cmca-12 to atomic I4 $/$ amd. Therefore, the molecularto-atomic phase transition is fairly isotope independent.

We find that our DMC results are essentially independent of the exchange-correlation (XC) functional used to calculate the orbitals for the trial wave function. However, the value of the proton $\mathrm{ZP}$ energy depends on the choice of $\mathrm{XC}$ functional. To investigate the effect of this, we have recalculated the harmonic ZP enthalpy for the $\mathrm{Cmca}-4$, Cmca-12, and $\mathrm{I}_{1} /$ amd structures using the Becke-LeeYang-Parr XC functional [55], as detailed in the Supplemental Material [39], which gives significantly different results from the PBE functional at the static lattice level. The phase diagram including the effects of the $\mathrm{ZP}$ harmonic enthalpy calculated with the Becke-Lee-YangParr functional leads to a reduction of $28 \mathrm{GPa}$ in the transition pressure for hydrogen dissociation, compared to the PBE-based phase diagram. The differences in dissociation pressure due to the flavor of $\mathrm{XC}$ functional used for the treatment of atomic vibrations do not affect the qualitative results presented in this work and only have a limited quantitative effect.

In conclusion, we have studied the dissociation of solid molecular hydrogen at the static lattice and dynamical lattice levels. At the static lattice level, our calculations give a transition from the $\mathrm{Cmca}-12$ molecular phase to the Cmca-4 molecular phase at $P=431 \mathrm{GPa}$, and a transition to the $I 4_{1} /$ amd atomic phase at $465 \mathrm{GPa}$. At the dynamical level, the molecular $\mathrm{Cmca}-12$ phase transforms directly to the atomic I4/ /amd phase at $374 \mathrm{GPa}$. The limited precision of our calculations prevents us from stating categorically that the $\mathrm{Cmca}-4$ phase does not exist, but the pressure range over which it might exist is very narrow. The atomization pressure is close to being within range of DAC experiments [56]. Therefore, the low-temperature molecular-to-atomic phase transition of high-pressure hydrogen might be observable experimentally. By comparing the dynamical phase diagrams of hydrogen and deuterium, we predict that the molecular-to-atomic phase transition is almost isotope independent. The proton $\mathrm{ZP}$ vibrational energies increase with pressure, and the anharmonic contribution leads to an increase in the vibrational energy of the molecular $\mathrm{Cmca}-4$ and $\mathrm{Cmca}-12$ phases and a decrease in that of the $I 4_{1} /$ amd atomic phase. Our results suggest that quantum melting of hydrogen would occur at finite temperature. Since metallic hydrogen is thought to be present in large amounts in the interiors of Jupiter, Saturn, and some extrasolar planets, planetary models should consider incorporating our prediction of the existence of an atomic metallic state at lower pressures than previously assumed.

We acknowledge the financial support of the U.K. Engineering and Physical Sciences Research Council under Grants No. EP/I030190/1 and No. EP/I030360/1, the use of 
the HECToR computing facilities (the U.K. national supercomputing service), the Imperial College London High Performance Computing Centre, and the Cambridge High Performance Computing Service. We acknowledge support from the Thomas Young Centre under Grant No. TYC-101.

*s.azadi@imperial.ac.uk

†bm418@cam.ac.uk

[1] E. Wigner and H. B. Huntington, J. Chem. Phys. 3, 764 (1935).

[2] J. M. McMahon, M. A. Morales, C. Pierleoni, and D. M. Ceperley, Rev. Mod. Phys. 84, 1607 (2012).

[3] A. F. Goncharov, R. T. Howie, and E. Gregoryanz, Low Temp. Phys. 39, 402 (2013).

[4] N. W. Ashcroft, Phys. Rev. Lett. 21, 1748 (1968).

[5] N. W. Ashcroft, Phys. Rev. Lett. 92, 187002 (2004).

[6] E. Babaev, A. Sudbo, and N. W. Ashcroft, Nature (London) 431, 666 (2004).

[7] M. I. Eremets and I. A. Troyan, Nat. Mater. 10, 927 (2011).

[8] R. T. Howie, C. L. Guillaume, T. Scheler, A. F. Goncharov, and E. Gregoryanz, Phys. Rev. Lett. 108, 125501 (2012).

[9] R. T. Howie, T. Scheler, C. L. Guillaume, and E. Gregoryanz, Phys. Rev. B 86, 214104 (2012).

[10] C.-S. Zha, Z. Liu, and R. J. Hemley, Phys. Rev. Lett. 108, 146402 (2012).

[11] P. Loubeyre, F. Occelli, and P. Dumas, Phys. Rev. B 87, 134101 (2013).

[12] C.-s. Zha, Z. Liu, M. Ahart, R. Boehler, and R. J. Hemley, Phys. Rev. Lett. 110, 217402 (2013).

[13] C. J. Pickard, M. Martinez-Canales, and R. J. Needs, Phys. Rev. B 85, 214114 (2012); 86, 059902(E) (2012).

[14] R. T. Howie, E. Gregoryanz, and A. F. Goncharov, J. Appl. Phys. 114, 073505 (2013).

[15] D. M. Ceperley and B. J. Alder, Phys. Rev. Lett. 45, 566 (1980).

[16] W. M. C. Foulkes, L. Mitas, R. J. Needs, and G. Rajagopal, Rev. Mod. Phys. 73, 33 (2001).

[17] C. Attaccalite and S. Sorella, Phys. Rev. Lett. 100, 114501 (2008).

[18] V. Natoli, R. M. Martin, and D. M. Ceperley, Phys. Rev. Lett. 70, 1952 (1993).

[19] S. Deemyad and I. F. Silvera, Phys. Rev. Lett. 100, 155701 (2008).

[20] M. I. Eremets and I. A. Trojan, JETP Lett. 89, 174 (2009).

[21] N. Subramanian, A. F. Goncharov, V. V. Struzhkin, M. Somayazulu, and R. J. Hemley, Proc. Natl. Acad. Sci. U.S.A. 108, 6014 (2011).

[22] S. Bonev, E. Schwegler, T. Ogitsu, and G. Galli, Nature (London) 431, 669 (2004).

[23] I. Tamblyn and S. A. Bonev, Phys. Rev. Lett. 104, 065702 (2010).

[24] J. Chen, X.-Z. Li, Q. Zhang, M. I. J. Probert, C. J. Pickard, R. J. Needs, A. Michaelides, and E. Wang, Nat. Commun. 4, 2064 (2013).

[25] B. Monserrat, N. D. Drummond, and R. J. Needs, Phys. Rev. B 87, 144302 (2013).

[26] J. P. Perdew, K. Burke, and M. Ernzerhof, Phys. Rev. Lett. 77, 3865 (1996).
[27] C. J. Pickard and R. J. Needs, J. Phys. Condens. Matter 23, 053201 (2011).

[28] Sam Azadi, W. M. C. Foulkes, and T. D. Kuhne, New J. Phys. 15, 113005 (2013).

[29] M. A. Morales, J. M. McMahon, C. Pierleoni, and D. M. Ceperley, Phys. Rev. B 87, 184107 (2013); Phys. Rev. Lett. 110, 065702 (2013).

[30] K. A. Johnson and N. W. Ashcroft, Nature (London) 403, 632 (2000).

[31] C. J. Pickard and R. J. Needs, Nat. Phys. 3, 473 (2007).

[32] J. M. McMahon and D. M. Ceperley, Phys. Rev. Lett. 106, 165302 (2011).

[33] S. Azadi and W. M. C. Foulkes, Phys. Rev. B 88, 014115 (2013).

[34] R. E. Cohen, Ivan I. Naumov, and Russell J. Hemley, Proc. Natl. Acad. Sci. U.S.A. 110, 13757 (2013).

[35] R. J. Needs, M. D. Towler, N. D. Drummond, and P. López Ríos, J. Phys. Condens. Matter 22, 023201 (2010).

[36] P. Giannozzi et al., J. Phys. Condens. Matter 21, 395502 (2009).

[37] The $\mathrm{Cmca}-12$ phase is metallic in the LDA but insulating up to $373 \mathrm{GPa}$ in $G W$ calculations. To check the accuracy of our metallic LDA-based trial functions for $\mathrm{Cmca}$-12, we also used trial functions from hybrid PBE0 calculations with a much larger band gap. The difference between the two DMC energies was less than $2 \mathrm{meV}$ per proton at all pressures considered.

[38] S. Azadi, C. Cavazzoni, and S. Sorella, Phys. Rev. B 82, 125112 (2010).

[39] See Supplemental Material at http://link.aps.org/supplemental/ 10.1103/PhysRevLett.112.165501 for technical details of the calculations.

[40] D. Alfè and M. J. Gillan, Phys. Rev. B 70, 161101 (2004).

[41] C. J. Umrigar, K. G. Wilson, and J. W. Wilkins, Phys. Rev. Lett. 60, 1719 (1988).

[42] N. D. Drummond and R. J. Needs, Phys. Rev. B 72, 085124 (2005).

[43] C. Lin, F. H. Zong, and D. M. Ceperley, Phys. Rev. E 64, 016702 (2001).

[44] S. Chiesa, D. M. Ceperley, R. M. Martin, and M. Holzmann, Phys. Rev. Lett. 97, 076404 (2006).

[45] N. D. Drummond, R. J. Needs, A. Sorouri, and W. M. C. Foulkes, Phys. Rev. B 78, 125106 (2008).

[46] P. López Ríos, A. Ma, N. D. Drummond, M. D. Towler, and R. J. Needs, Phys. Rev. E 74, 066701 (2006).

[47] B. Monserrat, N. D. Drummond, C. J. Pickard, and R. J. Needs, Phys. Rev. Lett. 112, 055504 (2014).

[48] Joon O. Jung and R. Benny Gerber, J. Chem. Phys. 105, 10 332 (1996).

[49] Joel M. Bowman, J. Chem. Phys. 68, 608 (1978).

[50] S. J. Clark, M. D. Segall, C. J. Pickard, P. J. Hasnip, M. I. J. Probert, K. Refson, and M. C. Payne, Z. Kristallogr. 220, 567 (2005).

[51] F. Lindemann, Phys. Z. 11, 609 (1910).

[52] P. A. Whitlock, D. M. Ceperley, G. V. Chester, and M. H. Kalos, Phys. Rev. B 19, 5598 (1979).

[53] S. T. Chui, Phys. Rev. B 41, 796(R) (1990).

[54] C. A. Burns and E. D. Isaacs, Phys. Rev. B 55, 5767 (1997).

[55] C. Lee, W. Yang, and R. G. Parr, Phys. Rev. B 37, 785 (1988).

[56] L. Dubrovinsky, N. Dubrovinskaia, V. B. Prakapenka, and A. M. Abakumov, Nat. Commun. 3, 1163 (2012). 